\title{
Postnatal Development of a Segmental Switch Enables Corticospinal Tract Transmission to Spinal Forelimb Motor Circuits
}

\author{
Samit Chakrabarty ${ }^{1,4}$ and John H. Martin ${ }^{1,2,3,4}$ \\ Departments of ${ }^{1}$ Neuroscience and ${ }^{2}$ Neurological Surgery and Psychiatry, Columbia University College of Physicians and Surgeons, New York, New York \\ 10032, ${ }^{3}$ New York State Psychiatric Institute, New York, New York 10032, and ${ }^{4}$ Department of Physiology and Neuroscience, City College of City University \\ of New York, New York, New York 10031
}

Development of skilled movements and the corticospinal tract (CST) begin prenatally and continue postnatally. Because the CST is required for skilled movements in maturity, it is accepted that motor skills cannot occur until the CST develops a mature organization. We recently showed that the CST plays an essential role in postnatal development of interneurons comprising the spinal circuits it engages. We proposed that CST signals are more effectively transmitted to ventral motor circuits after interneuron maturation, thereby enabling expression of CST motor functions, suggesting development of a segmental switch promoting transmission. We tested this by recording CST-evoked focal synaptic potentials, extracellularly, in the cervical enlargement of cats before and after interneuron maturation [postnatal week 5 (PW5) to PW7]. We compared monosynaptic CST amplitude input to segmental circuits with oligosynaptic ventral horn responses, as a measure of CST-evoked segmental response transmission from input to output. The M1 primary motor cortex was unilaterally inactivated between PW5 and PW7 to determine activity dependence. CST interneuron contacts were identified using confocal microscopy. CST terminals contact diverse interneuron classes. CST stimulation strongly activated ventral motor circuits at the ages when both interneurons and CST spinal terminations have developed a mature phenotype, supporting development of segmental transmission of CST signals. CST activity blockade impeded development of effective segmental transmission by the inactivated CST and created a novel path for transmission from the ipsilateral, unaffected, CST. Our findings show that development of segmental CST signal transmission regulates nascent CST motor control functions and provide insight into systems-level mechanisms for protracted motor skill development.

\section{Introduction}

Development of skilled motor behavior begins before birth with the in utero expression of interjoint movements and continues during a protracted postnatal period (Prechtl, 1997). The corticospinal (CS) system develops over a similarly protracted period (Martin et al., 2009). Because the corticospinal tract (CST) is required for skilled movements in maturity (Porter and Lemon, 1993), it is accepted that the expression of motor skills during development cannot occur until the CST achieves requisite motor milestones.

The immature CS system has several characteristics limiting skilled motor performance (Martin et al., 2009). In cats, at postnatal week 4 (PW4), the primary motor cortex (M1) map is absent and CST spinal terminations have an extensive immature regional distribution. At PW8, the motor map begins to be ex-

\footnotetext{
Received 0ct. 26, 2009; revised Dec. 7, 2009; accepted Dec. 13, 2009.

This work was supported by National Institutes of Health Grant NS36835 (J.H.M.). We gratefully acknowledge the assistance of Xiu Li Wu for histochemistry and Girma Asfaw and Dr. M. Osman for veterinary care.

Correspondence should be addressed to Dr. John H. Martin, Department of Physiology, Pharmacology, and Neuroscience, Sophie Davis School of Biomedical Education, City College of City University of New York, Harris Hall, Room 203, 160 Convent Avenue, New York, NY 10031. E-mail:jmartin@ccny.cuny.edu.

DOI:10.1523/JNEUROSCI.5286-09.2010

Copyright $\odot 2010$ the authors $\quad 0270-6474 / 10 / 302277-12 \$ 15.00 / 0$
}

pressed and CST terminations are essentially eliminated from the ventral horn and superficial dorsal horn. CST motor control is delayed until there is a structured M1 motor representation and the capacity for selective CST access to restricted spinal motor circuits.

Although there is a clear temporal association between skilled movement and CST development, it is not known whether maturation of the spinal circuits that the CST engages is important for achieving motor skills. Because animals express spinal reflexes at early ages (Villablanca and Olmstead, 1979), it has been simplistically assumed that segmental circuits mature early. However, we recently reported a novel CST function, pointing to a spinal mechanism for protracted development (Chakrabarty et al., 2009a). The CST exerts an activity-dependent trophic influence over spinal circuit development between PW5 and PW7: with an active CST, interneurons within the major target field of the tract develop a cholinergic phenotype, permitting cholinergic activation of postsynaptic targets. From the perspective of cholinergic excitation in the ventral horn, this suggests development of a segmental switch during the 2 week period of refinement that promotes transmission of CS signals. Such an increase in transmission would enable M1 to begin to exert control. This correlates with the rapid increase in expression of motor skills (Barrett and Bateson, 1978). 
In the present study, we tested the developmental switch hypothesis. We recorded CST-evoked focal synaptic potentials (FSPs) in the cervical enlargement of cats before and after PW5PW7, in response to pyramidal tract (PT) stimulation. We used the shortest-latency FSP as a measure of monosynaptic CST input to segmental circuits and longer-latency oligosynaptic ventral horn responses as a measure of segmental motor outflow. Comparing ventral horn output relative to segmental input provides a measure of segmental transmission. M1 was unilaterally inactivated between PW5 and PW7 to determine activity dependence of development of CST segmental transmission. CST axon-interneuron contacts were identified using confocal microscopy.

We show that PT stimulation more strongly activates ventral motor circuits at the ages when interneurons have developed a mature cholinergic phenotype and when CST terminations have a mature organization, supporting effective segmental transmission of CS signals. CST activity blockade impedes development of ventral transfer of signals by the inactivated CST and creates a novel path from the ipsilateral, unaffected, CS system. Our findings show that development of segmental transmission is a potentially strong regulator of nascent CST motor control functions and provide insights into systems-level mechanisms for the protracted development of motor skills.

\section{Materials and Methods}

General methods. All cats used in this study (PW4, $n=4$; PW8, $n=4$; PW11-PW14, $n=5$ ) were obtained from an Association for Assessment and Accreditation of Laboratory Animal Care accredited supplier. All experiments were conducted with the approval of the New York State Psychiatric Institute and Columbia University Institutional Animal Care and Use Committee.

General surgical procedures. A mixture of acepromazine $(0.03 \mathrm{mg} / \mathrm{kg}$, i.m.) and ketamine hydrochloride $(32 \mathrm{mg} / \mathrm{kg}$, i.m.) was given to induce anesthesia. For all survival surgeries, animals were administered atropine $(0.04 \mathrm{mg} / \mathrm{kg}$, i.m.). Animals were given a broad-spectrum antibiotic at the time of surgery (cephalozine, $25 \mathrm{mg} / \mathrm{kg}$, i.m.) and an analgesic after surgery (burprenorphine, $0.03 \mathrm{mg} / \mathrm{kg}$, i.m.). Cats were intubated after anesthesia was induced and maintained in an areflexive condition during surgery using $1-2 \%$ isoflurane. The head was placed in a stereotaxic frame, and a craniotomy was made over the motor cortex for tracer injection or implantation of the inactivation apparatus (see below). Animals resumed nursing after recovery from anesthesia and were given supplemental milk (kitten milk replacer; PetAg) as needed to ensure adequate weight gain.

For spinal electrophysiological experiments, animals were anesthetized $(30 \mathrm{mg} / \mathrm{kg}$, i.m., ketamine; $0.6-0.8 \mathrm{mg} / \mathrm{kg}$, i.m., xylazine; maintained using ketamine infusion at $10-30 \mathrm{mg} \cdot \mathrm{kg}^{-1} \cdot \mathrm{h}-1$, i.v.), the head was placed in a stereotaxic frame, and a cervical (C) laminectomy was made, as in our previous studies (Meng and Martin, 2003; Meng et al., 2004). Animals were administered atropine $(0.04 \mathrm{mg} / \mathrm{kg}$, i.m. ) during the experiment. The T1 spinous process was clamped to stabilize the vertebral column. The pyramidal tract electrode (concentric, tip diameter of $2-3 \mu \mathrm{m}$, oriented in the sagittal plane; Microprobe Inc.) was placed in the caudal medulla between 200 and $500 \mu \mathrm{m}$ lateral from the midline just rostral to the caudal edge of the occipital bone, depending on the age of the animal (supplemental Fig. 1, available at www.jneurosci.org as supplemental material).

Cortical tracer injections. Two to 4 weeks before conducting the terminal electrophysiological experiment, selected animals were prepared for anterograde tracer injection into M1. Animal were anesthetized as described above and mounted in a stereotaxic head holder (David Kopf Instruments). Under aseptic conditions, a craniotomy was made over the lateral portion of the frontal lobe to expose the forelimb representation of motor cortex. We pressure injected (Picopump; World Precision Instruments) the anterograde tracers biotinylated dextran amine (BDA) $(10 \%$ in PBS; Invitrogen) into the forelimb area of $\mathrm{M} 1$ of one hemisphere and
Lucifer yellow-dextran amine (LY-DA) (1\% in PBS; Invitrogen) into $\mathrm{M} 1$ of the other hemisphere. We used a standard pattern of tracer injections into the lateral sigmoid gyrus (three injections; $300 \mathrm{nl}$ each; $1.5 \mathrm{~mm}$ below the pial surface; separated by 1.0 to $1.5 \mathrm{~mm}$ ).

M1 activity blockade. To block neuronal activity, the $\mathrm{GABA}_{\mathrm{A}}$ agonist muscimol (10 mM in sterile saline; Sigma) was continuously infused using an osmotic minipump ( $0.5 \mu \mathrm{l} / \mathrm{h}$; model 2002; Alzet) into the center of the motor cortex forelimb representation, located primarily in the lateral sigmoid gyrus (Chakrabarty and Martin, 2000), as in our previous studies (Martin et al., 1999; Friel and Martin, 2005). A 28 gauge hypodermic needle cannula (Alzet), beveled at the tip, was connected with vinyl tubing (size 4; Scientific Commodities) to the pump. Under aseptic conditions, a craniotomy was made over the forelimb area of M1. The cannula was inserted below the pial surface. The cannula was fixed to the skull with screws and dental acrylic cement. The osmotic pump was placed in a subcutaneous pocket between the shoulder blades. Neuronal activity was blocked in left M1 from PW5 to PW7. The osmotic pump delivered muscimol for 2 weeks. Electrophysiology experiments were conducted either 1 or 4 weeks after the end of the infusion period.

In previous studies, we have used a variety of histochemical markers to assess the spatial effect of muscimol. Using the metabolic marker cytochrome oxidase as an indication of cortical function, muscimol infusion produces a maximal reduction within a $2.5-3 \mathrm{~mm}$ radial patch of cortex at the infusion site and a lesser reduction for an additional 4-5 mm (Martin et al., 1999). This suggests that the muscimol infusion produced maximal inhibition within the center of the $\mathrm{M} 1$ forelimb representation and reduced inhibition over a larger territory that extended into area 6 rostrally and somatic sensory cortex caudally. A similar distribution of reduced staining of the calcium binding protein parvalbumin was produced by muscimol infusion (Friel et al., 2007). We showed previously that the infusion did not produce a lesion by comparing cell body density (Martin et al., 2000) and the distribution of neurofilament F (SMI-32) immunoreactivity (Friel et al., 2007). We also found that the medullary pyramid cross-sectional areas on each side and SMI-32 staining in the lateral spinal column on each side were not different (Martin et al., 1999; Friel and Martin, 2005).

Pyramidal tract stimulation and electrophysiological recording and data acquisition. The pyramidal tract was stimulated with a biphasic square pulse, $0.2 \mathrm{~ms}$ wide, generated using a constant-current stimulator (model 2100; A-M Systems). In all animals, we determined the dorsoventral extent of effective medullary sites for evoking the spinal potentials, similar to our previous studies (Meng and Martin, 2003; Meng et al., 2004). Marking lesions were made in all animals to verify final electrode placement used during stimulation. Stimulus threshold current $(\mathrm{T})$ varied in relation to the animal's age (Meng and Martin, 2003), from $\sim 30$ to 90 $\mu \mathrm{A}$. In all experiments, stimulation was adjusted to $1.5 \mathrm{~T}$ for evoking spinal responses, unless otherwise noted.

The cord dorsum potential (CDP) was recorded on the surface at the dorsal root entry zone at C6 using a silver ball electrode. Depth recordings were made using a glass micropipette $(2 \mathrm{~m}$ potassium citrate, tip diameter of $1.5-2 \mu \mathrm{m}$, resistance of $2-5 \mathrm{M} \Omega$ ), using conventional amplification and filtration $(<100 \mathrm{~Hz}$ and $>10 \mathrm{kHz})$ and digitized. The small pipette tip was required to record focal responses, affording recording unit activity. We attempted to filter this during recording and after processing. For FSP analysis, measurements were made after filtering the acquired data (low pass, $3 \mathrm{kHz}$ ). We typically made at least three mediolateral penetrations at the same rostrocaudal level (caudal C6 to C7): (1) one through the medial dorsal horn that passed into laminae 7 and 8; (2) in the lateral dorsal horn and the lateral motor pools, underlying the dorsal root entry zone; and (3) halfway between (beneath the dorsal root entry zone), from the dorsal horn, intermediate zone, and the lateral motor pools. Surface recordings were made over the dorsolateral funiculus at rostral C6. We recorded nominally at $500 \mu \mathrm{m}$ depth intervals at each mediolateral penetration site. The records all show positive up. Data were collected at a sampling rate of $100 \mathrm{kHz}$, in $25 \mathrm{~ms}$ epochs for spinal recordings. We examined responses to single or short trains of up to three stimulus pulses (3.33 ms interpulse interval). Spinal potentials were acquired using an analog-to-digital converter (Digidata; Molecular De- 
vices) at $25 \mathrm{kHz}$ per channel. We used the program Axograph X (Axograph Scientific) for the Apple Macintosh computer to measure the latency and amplitude of spinal responses. Responses to individual stimuli (or trains) were recorded. For measurements and presenting potentials (see Fig. 3), we constructed ensemble averages synchronized on the stimulus artifact.

Criteria for focal synaptic potentials. PT-evoked FSPs were defined as a deflection (negative or positive) in the extracellular record evoked by the stimulus that changed over distances of $\sim 250 \mu \mathrm{m}$ and began to return or returned to baseline by the end of the recording epoch (typically within $5-10 \mathrm{~ms}$ ). As the pipette penetrated into a responsive zone, the slope of the FSP increased, and, as it left the responsive zone, the slope decreased. FSPs reflect the rate of current flow in a local population of neurons affected by the stimulus (Lorente de Nó, 1947). We verified the consistency of the FSPs by comparing the ensemble averages with displays of raw responses to single stimulus presentations. Figure 1 shows representative depth recordings (black) with the corresponding ensemble average (red) in relation to the surface-recorded CDP (dark gray, volley component; light gray, first postsynaptic response). The potentials, for the three ages, are all small and, despite their size, show consistency of onsets and waveforms, confirming that the averaged response is an accurate estimate of the individual potentials. Two responses are shown in $A$, one recorded at $1000 \mu \mathrm{m}$ below the pial surface (A2) and the other at 2000 $\mu \mathrm{m}$ (A3). The dotted line shows baseline, and the arrows point to the onset of the FSPs. The depth recording in $B$ shows an initial response in the monosynaptic range and a later response that we term oligosynaptic (B2; see below for monosynaptic vs oligosynaptic criteria). Ensemble averaging captures the two responses. Figure $1 C$ shows a small FSP recorded in a PW11 animal. Despite nonstimulus-driven spiking raising background noise, the FSP is clearly evident on both the individual traces and the ensemble average. We also found that the mean amplitude of the response measured on individual raw traces corresponded closely to the averaged response. For example, in Figure 1 B2, the mean amplitude for the initial response was $0.126 \mathrm{mV}$ and for the second response was 0.288 $\mathrm{mV}$, or 2.29 times larger than the first. The amplitudes of these responses were significantly different $(t=2.11 ; p=0.0036)$. Thus, the averaged FSPs correspond closely to the individual stimulus data. An FSP is distinguished from a broad negativity, which is a stereotypic slow-wave response that had an indistinct onset, did not begin to return to baseline within the recording epoch (typically within 5-10 ms), and often remained relatively constant across multiple recording sites. Such negative responses have been reported in response to PT stimulation (Illert et al., 1976) and are of unknown origin.

We measured three types of FSPs based on the latency relative to the volley component of the CDP: (1) the first negative FSP, which occurred either during the CDP postsynaptic component or within $\sim 1 \mathrm{~ms}$ after the volley; (2) the negative FSP that immediately followed the first but within $2.5 \mathrm{~ms}$ of the volley; and (3) when there was no early FSP, the first negative FSP that occurred after the CDP but also within $2.5 \mathrm{~ms}$ after the volley. Because timing is relative to the segmental volley, all latencies are segmental latencies. As conventionally interpreted for both sensory afferents and descending projections to the cord (Illert et al., 1976; Edgley and Jankowska, 1987; Noga et al., 1995), we infer synaptic linkage based on segmental latency. FSPs occurring within $\sim 1 \mathrm{~ms}$ after the volley are considered monosynaptic (Noga et al., 1995). Responses in this segmental latency range typically occur during the postsynaptic component of the CDP. Responses with a segmental latency $>1.1 \mathrm{~ms}$ are considered non-monosynaptic. Disynaptic CS excitation of motoneurons in adult cats occurs with a segmental latency ranging between 1.4 and $2.1 \mathrm{~ms}$ (Illert et al., 1974). Considering this segmental latency range, we conservatively limited our time window for non-monosynaptic responses to be $>1.2 \mathrm{~ms}$ and no more than $2.5 \mathrm{~ms}$. These responses are termed oligosynaptic; they likely include disynaptic and trisynaptic responses as described by Noga et al. (1995). It is theoretically possible that oligosynaptic responses recorded in the dorsal horn and intermediate zone, in which the CST terminates, partly reflect delayed monosynaptic responses, either produced by PT neurons that have a slower conduction volley than those evoking the shorter-latency response or an intraspinal conduction delay (Edgley and Jankowska, 1987). Although we found potentially im-
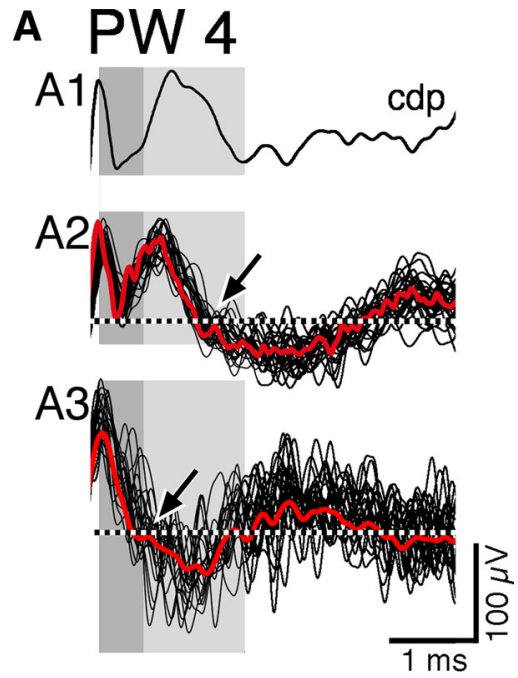

B PW 8

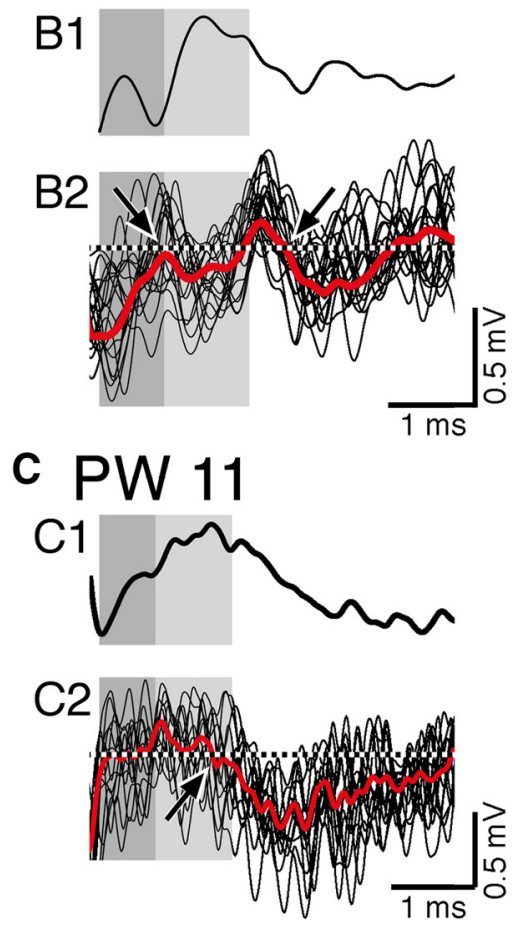

Figure 1. Raw and averaged FSPs. Representative examples of superimposed raw FSP recordings and corresponding ensemble average in relation to the surface-recorded CDP (top first trace in $\mathbf{A}-\mathbf{C}$ ). The dark gray shading indicates the volley component of the (DP, and the light gray shading indicates the initial postsynaptic component. The dotted lines are placed at 0 mV. A. Data from a PW4 animal. $\boldsymbol{A} 1$ shows the CDP, and $\boldsymbol{A} 2$ shows raw ( $n=27$; black traces) and averaged (red trace) depth recordings $1 \mathrm{~mm}$ from the pial surface. The arrow points to the onset of a negative FSP in the monosynaptic range. $A 3$ shows raw $(n=28)$ and averaged FSPs at a depth of $2 \mathrm{~mm}$. The arrow points to a negative FSP in the monosynaptic range. $\boldsymbol{B}$, Similar to $\boldsymbol{A}$ but for a PW8 animal and depth recordings $(n=25)$ only at $3 \mathrm{~mm}$. The left arrow points to the onset of a small FSP, and the right arrow points to a larger one. $C$, Similar to the other parts of the figure but for a PW11 animal and depth recordings $(n=23)$ only at $1 \mathrm{~mm}$.

portant developmental changes in the amplitude of late, presumably oligosynaptic, responses in the dorsal horn and intermediate zone, in the context of our development hypothesis, we only consider the late responses in the ventral horn. Importantly, it is highly unlikely that these FSPs are delayed monosynaptic responses because there are little or no CST projections to this region in the cat (Kuypers, 1981).

Analysis of focal synaptic potentials. We measured the onset latency, latency to peak negative response, and amplitudes of the responses rela- 
tive to both the onset of the response and to the prestimulus baseline (Fig. 1, dotted lines) on ensemble averages of FSPs. Statistical analyses revealed no significant difference between amplitudes measured in either way. We report only amplitudes relative to the onset of the response. For the purposes of localizing the FSPs, we used a simplified laminar distribution based on Rexed: (1) the dorsal horn was defined as Rexed's laminae 1-5; (2) the intermediate zone was defined as laminae 6 and the dorsal portion of lamina 7 (i.e., dorsal to the dorsal borders of laminae 8 and 9); and (3) the ventral horn was defined as recordings ventral to the laminae 8 and 9 dorsal borders). Figures $3-5$ show the laminae $5-6$ border and the borders of laminae 8 and 9.

Histology. At the end of the experiments, cats were deeply anesthetized (sodium pentobarbital, $30 \mathrm{mg} / \mathrm{kg}$, i.v.) and perfused transcardially with warm saline, followed by a solution of $4 \%$ paraformaldehyde [in $0.1 \mathrm{M}$ phosphate buffer $(\mathrm{PB}), 4^{\circ} \mathrm{C}, \mathrm{pH} 7.3-7.4 ; 1000 \mathrm{ml} / \mathrm{kg}$ body weight] at $\mathrm{pH}$ 7.4. Heparin was injected (200-500 U, i.v.) at the onset of perfusion. The brain and spinal cord were removed, postfixed in the same fixative at $4^{\circ} \mathrm{C}$ for $2-3 \mathrm{~h}$, and then transferred to $20 \%$ sucrose in PB overnight. Parasagittal sections through the cortex were cut, and alternate sections were processed for BDA or LY-DA to determine the location of tracer injection sites and Nissl stained for assessing cortical cytoarchitecture. Transverse sections were cut through the caudal medulla and through the region of the spinal cord examined. Tissue was Nissl stained and examined for the PT electrode lesion site and track left by the spinal electrode during penetrations and depth recording sites. Depths in the spinal cord were measured in relation to landmarks (i.e., gray matter borders).

Tracer histochemistry. For visualization of BDA, sections were incubated in PBS containing the avidin-biotin complex reagent as prescribed by the manufacturer (ABC kit; Vector Laboratories) and $0.2 \%$ Triton $\mathrm{X}-100$ for $2 \mathrm{~h}$ at room temperature (RT). After rinsing, sections were incubated with the chromogen diaminobenzidine (DAB) (Sigma) for 6-30 min. To examine the relation between BDA and interneuron markers, we used a fluorescent fluorophore. The sections were incubated with conjugated ExtrAvidin FITC (1:200; Sigma) or ExtrAvidin cyanine 3 (Cy3) (1:500 to 1:800; Sigma) overnight at $4^{\circ} \mathrm{C}$. After rinsing, sections were mounted on gelatin-coated slides, air dried overnight, and coverslipped. To visualize axons labeled with LY-DA, sections were incubated at $4^{\circ} \mathrm{C}$ overnight in PBS containing rabbit anti-LY-DA antibody (1:1000; Invitrogen) in blocking buffer ( $3 \%$ donkey serum in $1 \times$ PBS with $0.2 \%$ Tween 20, pH 7.4). After rinsing, sections were incubated for $2 \mathrm{~h}$ at RT in blocking buffer containing $0.2 \%$ anti-rabbit secondary antibody conjugated to peroxidase (1:200; $\mathrm{pH} 7.4)$. After rinsing, sections were incubated with the chromogen DAB for 5-30 min. As with BDA, we also used fluorescence to mark LY-DA. We either used a secondary antibody conjugated to Cy3 (1:800, incubated at RT for $1 \mathrm{~h}$; donkey anti-rabbit, antigoat, or anti-mouse) or to FITC (1:500, at RT for $2 \mathrm{~h}$; donkey anti-rabbit, anti-goat, or anti-mouse). We used a quantitative method for determining the topographic distribution of label within the gray matter in the cervical enlargement, as described previously (Brus-Ramer et al., 2007; Friel and Martin, 2007).

Calcium binding protein and choline acetyltransferase immunohistochemistry. We used immunohistochemistry to visualize the distribution of cells containing choline acetyltransferase (ChAT), calbindin (CB), parvalbumin (PV), and calretinin (CR), as in our previous study, in which the epitopes are reported (Chakrabarty et al., 2009a). The following antibodies were used, at the concentrations and incubation times indicated: (1) rabbit polyclonal anti-CB D-28K (Millipore Bioscience Research Reagents), 1:500, $4^{\circ} \mathrm{C}, 2 \mathrm{~d}$; (2) mouse monoclonal anti-PV (PV235; Swant), 1:5000, $4^{\circ} \mathrm{C}$, overnight; (3) mouse monoclonal anti-CR (MAB1568; Millipore Bioscience Research Reagents), 1:1000, $4^{\circ} \mathrm{C}$, overnight; and (4) goat polyclonal anti-ChAT (Millipore Bioscience Research Reagents), 1:100, $4^{\circ} \mathrm{C}$, overnight. For each primary antibody, freefloating sections were incubated at RT in PBS containing the primary antibody in blocking buffer ( $3 \%$ donkey serum in $1 \times$ PBS with $0.2 \%$ Tween 20, pH 7.4). After rinsing, sections were incubated at RT in blocking buffer containing secondary antibody conjugated to Cy3 (1:800 at RT for $1 \mathrm{~h}$; donkey anti-rabbit, anti-goat, or anti-mouse), FITC (1:500 at RT for $2 \mathrm{~h}$; donkey anti-rabbit, anti-goat, or anti-mouse), or peroxidase (1:200 at RT for $2 \mathrm{~h}$ ), at pH 7.4. For fluorescence labeling, sections were mounted on gelatin-coated slides, air dried, and coverslipped with Vectashield (Vector Laboratories). For peroxidase staining, sections were dehydrated before coverslipping.

Confocal microscopy. To determine whether CST axons contacted spinal cord neurons directly, we used laser-scanning confocal microscopy (LSM META510; Carl Zeiss) using two different fluorescent markers: FITC (488 nm excitation, $520 \mathrm{~nm}$ emission) and Cy3/rhodamine (550 $\mathrm{nm}$ excitation, $573 \mathrm{~nm}$ emission). For imaging FITC, the argon laser of the confocal microscope was set to excite the sample at $488 \mathrm{~nm}$, and the emitted light was filtered to transmit between 505 and $550 \mathrm{~nm}$. For Cy3, the helium-neon laser excited at $543 \mathrm{~nm}$, and the emitted light was filtered to transmit between 560 and $615 \mathrm{~nm}$. We also used deconvolution microscopy and a Carl Zeiss inverted Axioscope 200M using $z$-series image capture and deconvolution software (Volocity; Improvision) to obtain high-resolution thin optical sections. After deconvolution (eight runs using a measured point-spread function for the lens used), images of each $z$-plane were then merged. For epifluorescence, a 460-500 $\lambda$ excitation filter and a 510-560 $\lambda$ emission filter was used for FITC, and a 535-550 $\lambda$ excitation filter and a 610-675 $\lambda$ emission filter was used for Cy3. To adjust color balance, contrast, and brightness in the confocal images, NIH ImageJ and Adobe Photoshop (Adobe Systems) was used. When comparing images, all capture and adjustment parameters were kept identical.

Statistical analyses. Standard statistical tests including Student's $t$ test and ANOVA were conducted using JMP 7 (SAS Institute) and Statview. We used the Bonferroni's/Dunn's post hoc test for significance $(p<0.05)$ between groups.

\section{Results}

\section{CST axon terminations make direct contacts with identified} spinal interneuron classes

Whereas several previous studies have used the distribution of CST axon terminations to infer contacts with particular spinal neurons (Weidner et al., 2001; Chakrabarty et al., 2009a), none have used confocal techniques and thin optical slicing to demonstrate direct contacts. In this study, we used laser-scanning confocal microscopy to slice fixed transverse spinal $40 \mu \mathrm{m}$ sections optically into $1 \mu \mathrm{m}$ sections (Fig. $2 A-F$ ). Contacts with two different ChAT interneurons are shown in Figure 2, $A$ and $B$. At PW8 and older, CST axon terminations make abundant contacts with ChAT-positive interneurons within the central region of the deep dorsal horn and intermediate zone but, as we reported previously, before PW8 ChAT staining is weak within this region (Chakrabarty et al., 2009a). The main parts of Figure 2, $A$ and $B$, show projection images, and the insets show single $1 \mu \mathrm{m}$ optical slices. Representative examples of double-labeled CST boutons (i.e., tracer and ChAT), which contain yellow pixels, are shown on the cell body of ChAT interneurons as well as on the proximal dendrites (arrows). Figure 2, $C$ and D, shows representative examples of CST axon terminations (arrows) contacting the cell body of a PV-positive interneuron $(C)$ and a CB-positive interneuron $(D)$. These two interneuron classes are thought to be inhibitory (Kubota and Jones, 1993). Contacts on the cell body at two different depths are shown for the PV interneuron in $C$. Despite dense CST terminations within a field of CB interneurons and their processes in this representative PW4 animal, there were surprisingly few contacts. We found no contacts between CST axons and CB interneurons in kittens 8 weeks and older. In contrast, CST contacts on CB-positive interneurons were more common in older kittens after previous CST inactivation (Fig. $2 G$; discussed below). Figure 2, $E$ and $F$, shows representative examples of CST contacts on the cell body of a large CR-positive interneuron located laterally in the intermediate zone $(E)$ and a smaller CR cell located laterally ( $F$; optical slices at two depths are shown). These findings, together with previous topographic data 

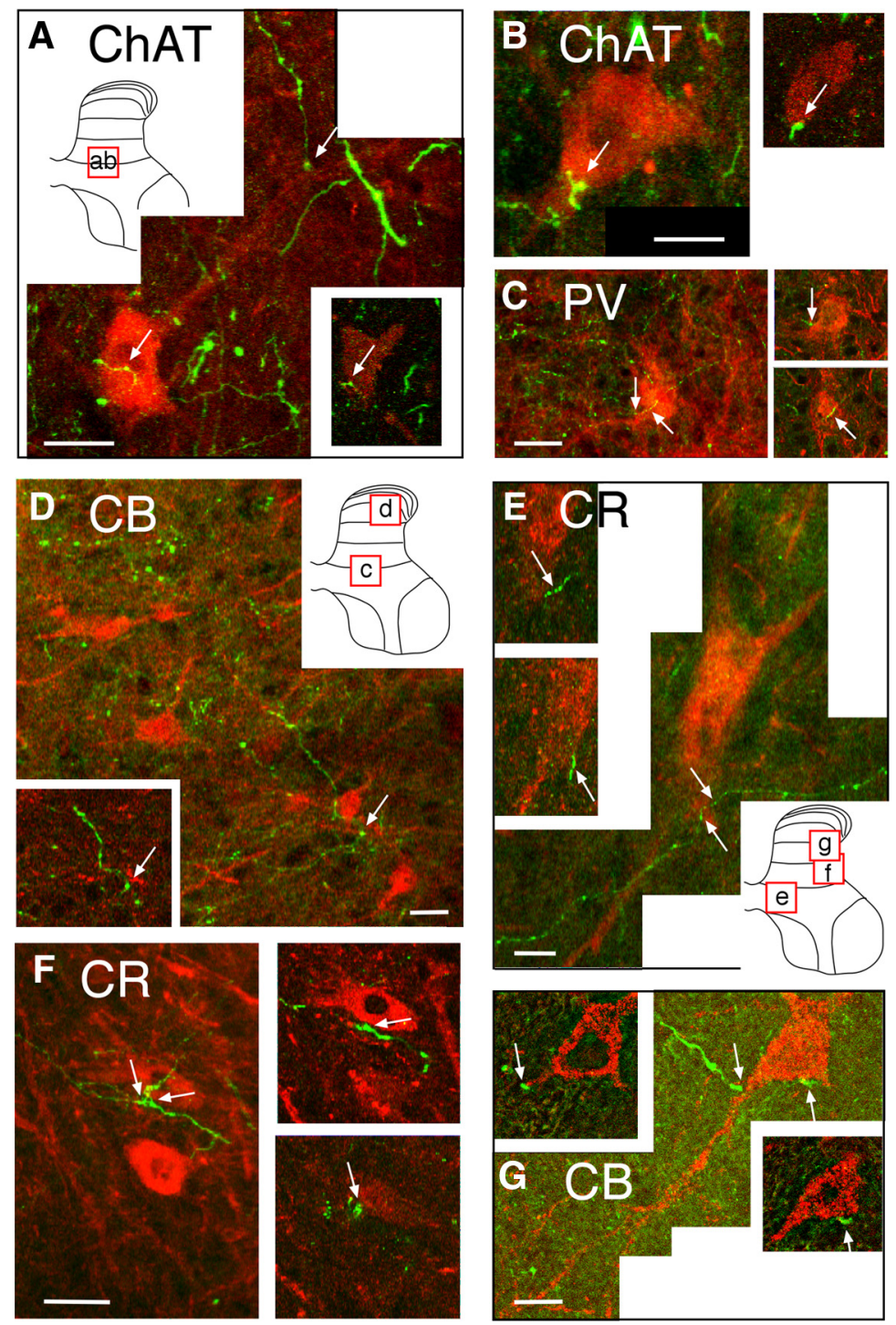

Figure 2. Confocal micrographs showing contact between CST axon terminals and spinal interneurons. Each lettered part shows a projection image, and the micrograph insets present a single $1 \mu \mathrm{m}$ optical slice. $\boldsymbol{A}, \boldsymbol{B}$, Two representative ChAT-positive interneurons (red) contacted by labeled (ST axons (green). Arrows in $A$ show two points of contact, one of which (on cell body) is shown on the $1 \mu \mathrm{m}$ optical slice (inset, bottom right). Arrow on projection image in $\boldsymbol{B}$ points to a contact on the cell body. The inset is a $1 \mu \mathrm{m}$ optical slice showing the same contact point. The locations of the projection images in $A$ and $B$ are shown in the schematic gray matter inset in $A$. C, PV-positive interneuron (red). The arrows on the projection image point to contact between a labeled CST axon and the cell body. The insets at the right show these contacts on two separate $1 \mu$ m optical sections at different $z$-axis depths. The location of the micrograph is shown in the inset in $\boldsymbol{D}$. $\boldsymbol{D}$, (B-positive interneurons (red). As in the other figures, the contact between the CST axons and an interneuron is marked by the arrow. $1 \mu \mathrm{m}$ optical slice through the contact is shown in the inset. The location of the cells is shown in the inset in $\boldsymbol{D} . \boldsymbol{E}, \boldsymbol{F}, \mathrm{CR}$-positive interneuron. The arrows on the projection image in $\boldsymbol{E}$ point to contact between a labeled CST axon and the cell body of one interneuron. The optical sections show the two marked contacts on the cell shown in the projection image. The projection image in $\boldsymbol{F}$ shows several contact points (arrows). The optical sections at the right are the contacts between the CST axons and the same calretinin-positive neuron. The contacts on this cell, as well as many others, are varicose portions of the axon, suggesting that they are boutons. The locations of the cells are shown in the inset in E. G, CB-positive interneuron from an animal that received unilateral $\mathrm{M} 1$ inactivation. Section taken from the side contralateral to inactivation (i.e., silenced or affected side of the spinal cord). Two points of contact are seen in the optical slices; only one of the contacts is shown on the projection image. $\mathbf{G}$ is a deconvolution image. The location of the cell is shown in the inset in $\boldsymbol{E}$. Scale bars, $2 \mu \mathrm{m}$.

showing the distribution of CST terminations in relation to particular interneuron classes (Chakrabarty et al., 2009a), are the first steps toward elucidating CST spinal motor circuits in the cat.

\section{Evoked contralateral monosynaptic and oligosynaptic CST} responses increase during normal postnatal development Monosynaptic FSPs (for FSP criteria, see Materials and Methods) were distinguished from oligosynaptic events on the basis of the

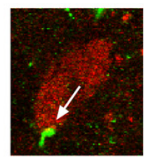

latency of a response evoked by a single PT stimulus in relation to the descending volley and the postsynaptic component of the CDP. The first CST-evoked response was termed the monosynaptic response if it occurred within $1 \mathrm{~ms}$ after the descending CDP volley (Noga et al., 1995) or the first deflection from baseline (in PW4). The oligosynaptic response we examined occurred later $(2.367 \pm 0.131 \mathrm{~ms})$ but closely corresponded to the time range of recorded evoked disynaptic EPSP latencies of 1.4-2.1 $\mathrm{ms}$ in motoneurons in barbiturate-anesthetized cats (Illert et al., 1974, 1976). The mean latency for monosynaptic responses in relation to the CDP volley at PW4 and PW8 were not different $(0.966 \pm 0.024$ and $0.940 \pm 0.065, p=0.7384)$. Monosynaptic responses at PW11 $(0.718 \pm 0.055 \mathrm{~ms})$ were significantly shorter than the other ages (ANOVA, three groups, $F=7.235, p=$ 0.0114; Bonferroni's/Dunn's post hoc; PW11 vs PW4, $p=0.0068$ significant $(\mathrm{S})$; PW11 vs PW8, $p=0.0125$ S; PW8 vs PW4, $p=0.7384$ ). For oligosynaptic responses, there was no difference across ages (ANOVA, $F=1.415, p=0.2877$ ).

Ketamine anesthesia eliminated reflexevoked responses but left the spinal cord in an excited state, with spontaneous FSPs and spontaneous unit activity. This contrasts with the barbiturate-anesthetized preparation, in which spinal excitability was very low, and these spontaneous responses were absent (S. Chakrabarty, unpublished observations). Spontaneous spinal activity (FSPs and unit activity) was most prominent in the middle electrode penetration at PW4 (Fig. 3B) and in all electrode penetrations in older animals, often resulting in a noisier baseline. Nevertheless, constraining our analyses to the monosynaptic and short-latency oligosynaptic range ensured that we were assessing specifically CST-evoked responses. The medial electrode penetration (Fig. 3A) shows an example of a monosynaptic FSP in the dorsal horn (dorsal horn boundary shown as thin gray line). In the intermediate zone, a negative response was recorded that remained at its peak value for the duration of the recording epoch $(2000 \mu \mathrm{m})$. Farther ventrally, shallow and broad negativities were recorded in the ventral horn (2500 and $3000 \mu \mathrm{m})$. Because of its stereotypic configuration and indistinct offset, these persistent negativities are not consistent with an FSP. Similar slow negative responses have been reported after PT stimulation (Illert et al., 1976). Occasionally, we recorded a focal positivity, as demonstrated in the third trace in this penetration (Fig. $3 A, 1500 \mu \mathrm{m}$ ). The peak of the positive response coincided with the peak negativity in the next deeper recording, suggesting that this could be a current source for the deeper negativity. The positive response could, 

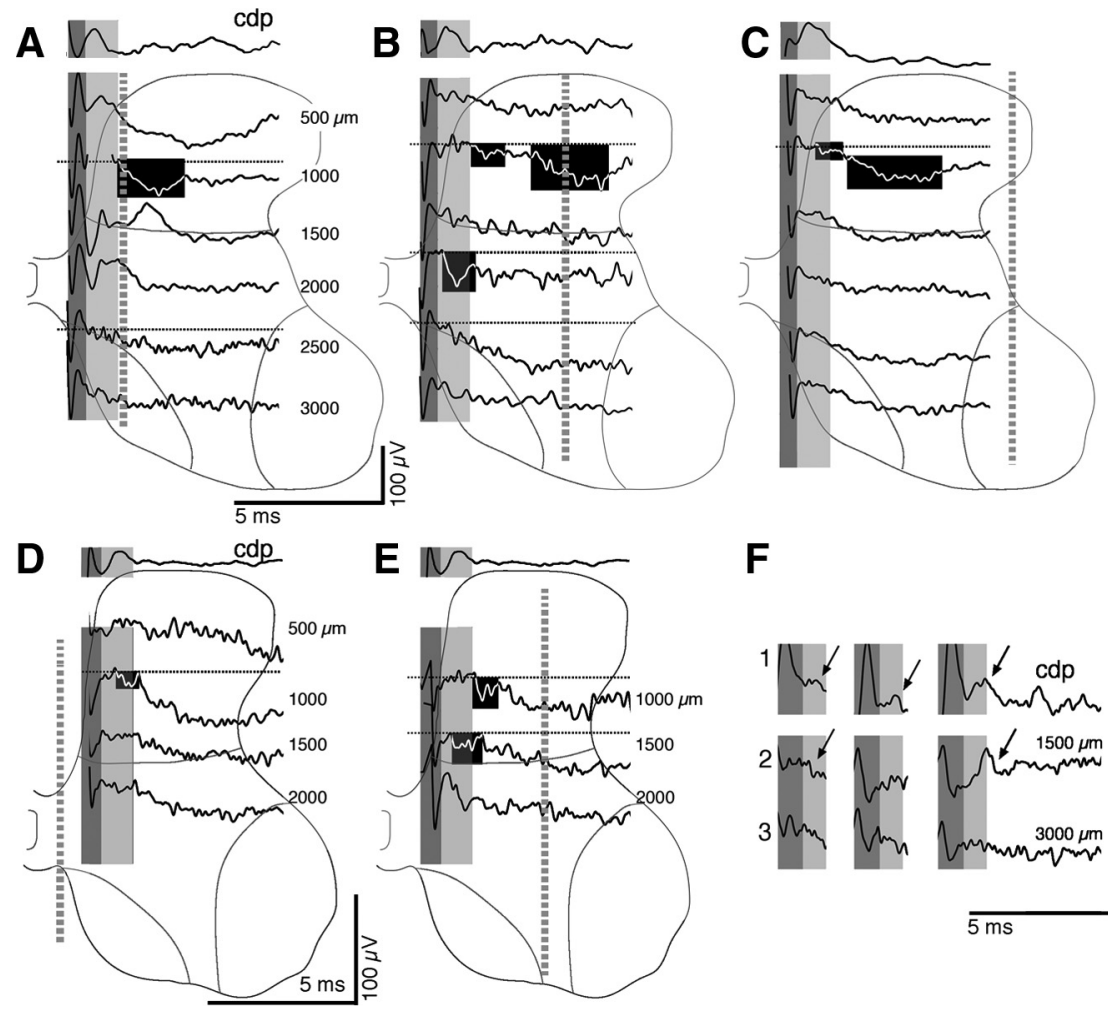

$\mathbf{F}$

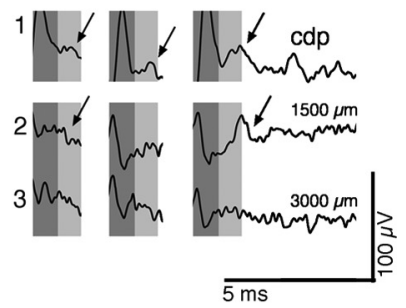

Figure 3. Focal synaptic potentials at PW4. A-E show the CDP (top traces) and associated field recordings at the depths indicated (in micrometers) and by the spinal gray matter outlines. The border between Rexed's laminae 5 and 6 is shown, as well as the borders of laminae 8 and 9 . The dark gray bands mark the volley, and the light gray bands indicate the time of the CDP postsynaptic response. The blackened regions highlight examples of a range of small and large FSPs in the monosynaptic (beginning during the CDP postsynaptic response) and oligosynaptic (for description, see Results) ranges. The electrode penetrations are shown as gray dashed lines. Ventral horn responses correspond to the depths in which recording were made within laminae 8 and 9 or between these laminae, within deep lamina 7. Broad negativities were recorded at several sites. As indicated in Results, these responses typically had indistinct onsets and did not recover to baseline during the $5-10 \mathrm{~ms}$ after stimulus recording period; we have not included these responses as FSPs (see Materials and Methods). Dotted lines in $\boldsymbol{A}-\boldsymbol{F}$ indicate baseline. $\boldsymbol{A}-\boldsymbol{C}$ are from the same animal: $\boldsymbol{A}$, along the medial gray matter border; $\boldsymbol{B}$, under the dorsolateral funiculus; $\boldsymbol{C}$, laterally in the gray matter. The penetrations shown in $\boldsymbol{D}$ (close to the midline) and $\boldsymbol{E}$ (under the dorsolateral funiculus) are from a different animal. $\boldsymbol{F}$ shows facilitation of the CDP and intermediate zone but not deep ventral horn FSP. In this test, stimulation was at three times threshold, with three pulses separated by $3.33 \mathrm{~ms} . \mathbf{1}$, CDP; 2 , an intermediate zone recording and a ventral horn recording (3), from the animal shown in $\boldsymbol{A}-\mathbf{C}$. The arrows highlight a facilitating postsynaptic CDP response (1) and focal negativity (2) in the intermediate zone.

however, be a disynaptic inhibitory response because its onset is slightly delayed compared with the nearby negativities. The middle electrode penetration (Fig. $3 B$ ) showed a small, phasic, and highly localized FSP in the monosynaptic range in the upper dorsal horn $(1000 \mu \mathrm{m})$. Its onset was just after the end of the postsynaptic component of the CDP but within our monosynaptic latency criterion. An especially large and phasic monosynaptic response was recorded in the intermediate zone $(2000 \mu \mathrm{m})$. As with the medial electrode penetration, the middle recording revealed only a broad and indistinct negativity in the ventral horn $(2500 \mu \mathrm{m})$ that flattened at the most ventral recording depth. Laterally (Fig. $3 C$ ), there was minimal evoked activation. Superficially and ventrally, only broad negativities were recorded. In this example, small responses were recorded only in the deeper dorsal horn $(1000 \mu \mathrm{m})$. Figure $3 D$ shows recordings from another PW4 animal in which the medial electrode penetration traversed the most medial portion of lamina 7 , in which there is a small cluster of ChAT-positive interneurons (Barber et al., 1984; Miles et al., 2007; Chakrabarty et al., 2009a; Zagoraiou et al., 2009). We previously found that the CST does not project to this region until after PW8 (Chakrabarty et al., 2009a). Interestingly, recordings at the level of the central canal (Fig. 3D, ventral two traces, 1500 and $2000 \mu \mathrm{m}$ ) failed to record a FSP, whereas immediately dorsal (Fig. 3D, second trace, highlighted, $1000 \mu \mathrm{m}$ ) and more lateral (Fig. $3 E, 1000$ and $1500 \mu \mathrm{m}$ ) recordings in the same animal showed clear responses in the monosynaptic range.

The lack of ventral horn FSPs in response to PT stimulation at 4 weeks was also observed with multiple pulses of stronger stimuli $(3 \mathrm{~T}$, three times, 333 $\mathrm{Hz}$ ). Figure $3 \mathrm{~F} 2$ shows an example of a response recorded from the intermediate zone with three stimuli at $3 \mathrm{~T}$. Whereas multiple stimulation resulted in a small amount of facilitation of the CDP (Fig. $3 F 1$, arrows) and the monosynaptic response (Fig. 3F2, arrow), there was no ventral oligosynaptic potential (Fig. 3F3). To summarize, at PW4, FSPs were commonly recorded within regions of the dorsal horn and intermediate zone but not in the ventral horn.

In PW8 and older animals, FSPs were substantially larger than at PW4. It should be noted that recordings in animals at PW8 and older were collected at $1 / 10$ the gain as those in PW4 animals. In contrast to PW4, responses in PW8 and older animals were present ventrally along with dorsal and intermediate sites. Figure $4 \mathrm{~A}$ shows responses from one animal, and Figure 4, $B$ and $C$, shows those from a different animal. Monosynaptic responses were present throughout the dorsal horn and intermediate zone (highlighted in $A-C)$. Importantly, oligosynaptic responses were now recorded ventrally (highlighted in Fig. 4B,C). By PW11, we recorded robust monosynaptic responses in all electrode penetrations (Fig. 5). The medial penetration (Fig. 5A) shows examples of two responses with a rapid time-to-peak (at 1500 and 2000 $\mu \mathrm{m})$. This is a region with particularly dense CST terminations (Li and Martin, 2001). Other examples are shown in the middle and lateral penetrations (Fig. $5 B, C$ ). At this age, phasic oligosynaptic responses were commonly observed ventrally. For example, there was a phasic deep oligosynaptic field in the middle penetration (Fig. $5 B$, highlighted, $3500 \mu \mathrm{m}$ ) that was not preceded by a monosynaptic field. Examining raw traces revealed consistent and sharp onsets and peaks with each stimulus. Similarly, Figure $5 C$ shows evidence of another phasic ventral field, beginning after a clear transition from an earlier monosynaptic FSP (highlighted, $3500 \mu \mathrm{m})$. By PW8 and older, CST terminations are pruned dorsally and ventrally (Li and Martin, 2000, 2001). Despite the absence of ventral CST projections at PW8 and PW11, ventral oligosynaptic responses are more prominent than at PW4, suggesting that CST inputs to the dorsal horn and intermediate zone can more effectively activate ventral motor circuits, leading to a motor output. 

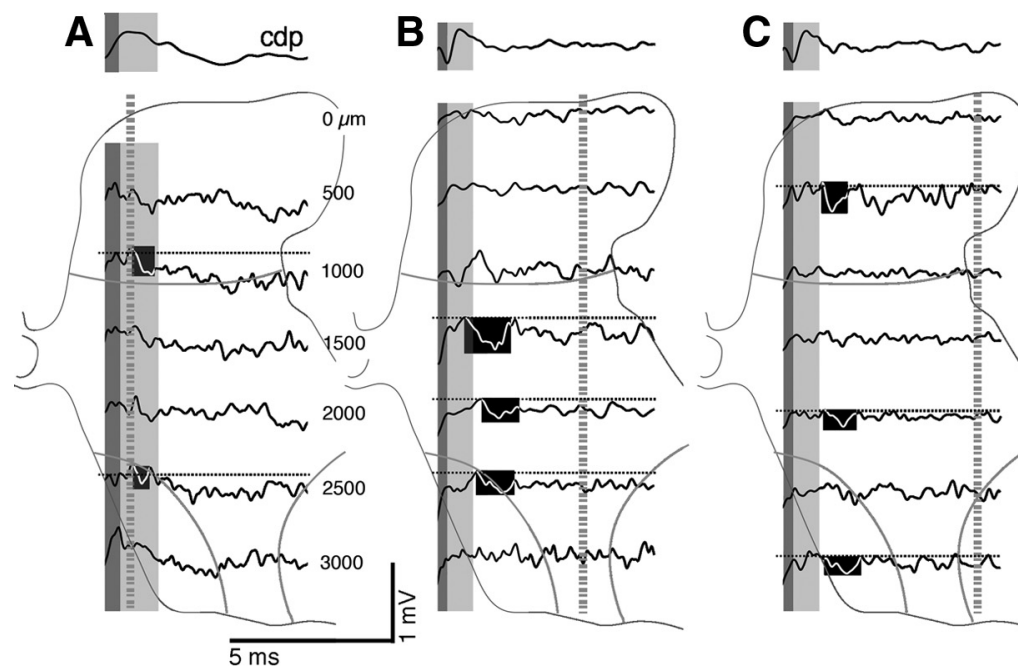

Figure 4. Focal synaptic potentials at PW8. Same format as Figure $3 A-C$, with the CDP recordings above the depth recordings. The bottom two traces in each component correspond to ventral horn responses (i.e., at depths in which recording were made within or between laminae 8 and 9). The blackened regions highlight examples of small and large FSPs in the monosynaptic (beginning during the CDP postsynaptic response) and oligosynaptic ranges.
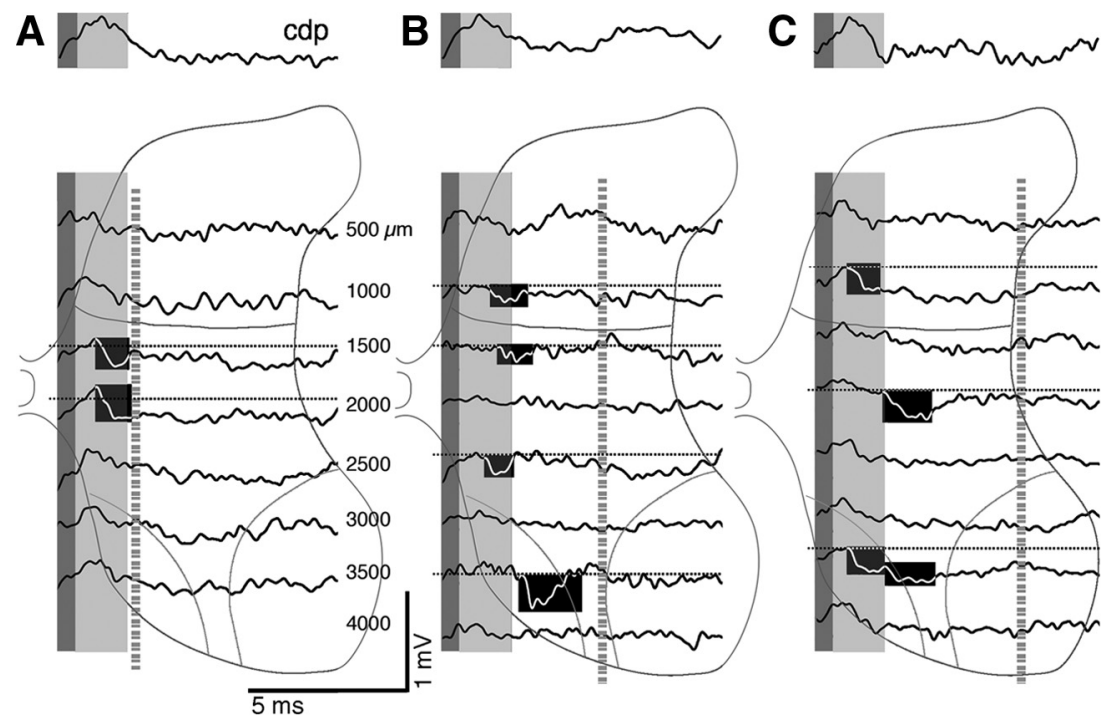

Figure 5. Focal synaptic potentials at PW11. Same format as Figures $3 A-C$ and 4 . The bottom three traces in each component correspond to ventral horn responses (i.e., at depths in which recordings were made within or between laminae 8 and 9 ). The blackened regions highlight examples of small and large FSPs in the monosynaptic (beginning during the CDP postsynaptic response) and oligosynaptic ranges. Dotted horizontal lines indicate baseline.

\section{CST segmental signal transmission is limited before PW8}

We measured the amplitude of the maximal monosynaptic and oligosynaptic FSP in each animal. For the dorsal horn and intermediate zone, we chose the largest potential, regardless of the mediolateral location of the electrode penetration, because the CST terminates throughout these regions. For the ventral horn, the largest potentials typically occurred within either the middle or lateral electrode penetrations, close to or within the lateral motor pools. Figure 6 shows the results of this analysis. Responses in the monosynaptic range (Fig. $6 \mathrm{~A}$ ) in the dorsal horn and intermediate zone showed a strong age dependence, whereas ventral horn monosynaptic responses remained small (dorsal horn: $F=9.298$, $p=0.0003 ; 11$ and 4 weeks, $p=0.0123 \mathrm{~S} ; 11$ and 8 weeks, $p=$ $0.3751 ; 4$ and 8 weeks, $p=0.0720$; intermediate zone: $F=8.635$, $p=0.0066 ; 11$ and 4 weeks, $p=0.0021 \mathrm{~S} ; 11$ and 8 weeks, $p=$
$0.2499 ; 4$ and 8 weeks, $p=0.0211$ S; ventral horn: $F=1.9, p=0.2192$ ). Oligosynaptic responses also showed age dependence (dorsal horn: $F=7.154, p=$ $0.0138 ; 11$ and 4 weeks, $p=0.0015 \mathrm{~S} ; 11$ and 8 weeks, $p=0.1010 ; 4$ and 8 weeks, $p=0.0373$; intermediate zone: $F=$ $13.346, p=0.0020 ; 11$ and 4 weeks, $p=$ 0.0002 S; 11 and 8 weeks, $p=0.0561 ; 4$ and 8 weeks, $p=0.0056$; ventral horn: $F=$ 23.966, $p=0.0002$; 11 and 4 weeks, $p=$ $0.0001 \mathrm{~S} ; 11$ and 8 weeks, $p=0.0013 \mathrm{~S} ; 4$ and 8 weeks, $p=0.0514$ ). Importantly, there was a disproportionate increase in the ventral horn oligosynaptic response (Fig. 6B, VH). For the monosynaptic responses at all dorsoventral levels and oligosynaptic responses in the dorsal horn and intermediate zone, the increase in peak amplitude was much larger between 4 and 8 weeks than 8 weeks and older. In contrast, the increase in the ventral horn oligosynaptic response was very large from 4 to 8 weeks but larger still for 8 weeks and older.

The dorsal horn and intermediate zone are the principal termination sites for the CST in the cat (Martin, 1996). There are no projections in the motor pools, and the projection to the ventral portion of lamina 7 , between laminae 8 and 9, is very sparse (Li and Martin, 2000; Chakrabarty et al., 2009). We used the ratio of the ventral horn oligosynaptic and the monosynaptic dorsal horn and intermediate zone responses as a measure of segmental CST transmission (i.e., output/input). Figure $6 C$ plots these ratios. At PW4, the ratios are $<1$. Small dorsal horn and intermediate zone monosynaptic responses precede an even smaller ventral horn oligosynaptic response. At PW8, the ratios are $>1$ (1.36 and 1.21). These are significantly greater than at PW4. In the older animals, the ratios are even greater (2.97 and 2.56). The largest increase is between PW4 and PW8 (6-fold for oligosynaptic ventral horn/ monosynaptic dorsal horn, 5.67-fold for oligosynaptic ventral horn/monosynaptic intermediate zone) (oligosynaptic ventral horn/dorsal horn, ANOVA, $F=19.812$, $p=0.0003 ; 11$ and 4 weeks, $p<0.0001 \mathrm{~S} ; 11$ and 8 weeks, $p=$ $0.0045 \mathrm{~S} ; 4$ and 8 weeks, $p=0.0351$; oligosynaptic ventral horn/ monosynaptic intermediate zone, ANOVA, $F=20.909, p=$ $0.0003 ; 11$ and 4 weeks, $p<0.0001 \mathrm{~S} ; 11$ and 8 weeks, $p=0.0043$ S; 4 and 8 weeks, $p=0.0274$ ). This is the period that CST axons achieve a mature termination pattern and target regions in the intermediate zone develop the mature ChAT phenotype. These findings show that there is limited transmission from the CST into ventral spinal motor circuits before the CST refines its segmental terminations, whereas after refinement (and ventral terminations are eliminated) (Li and Martin, 2000), transmission is effective.

Although injury discharge and intermittent spiking was present throughout all recordings in all animals, we noticed that 

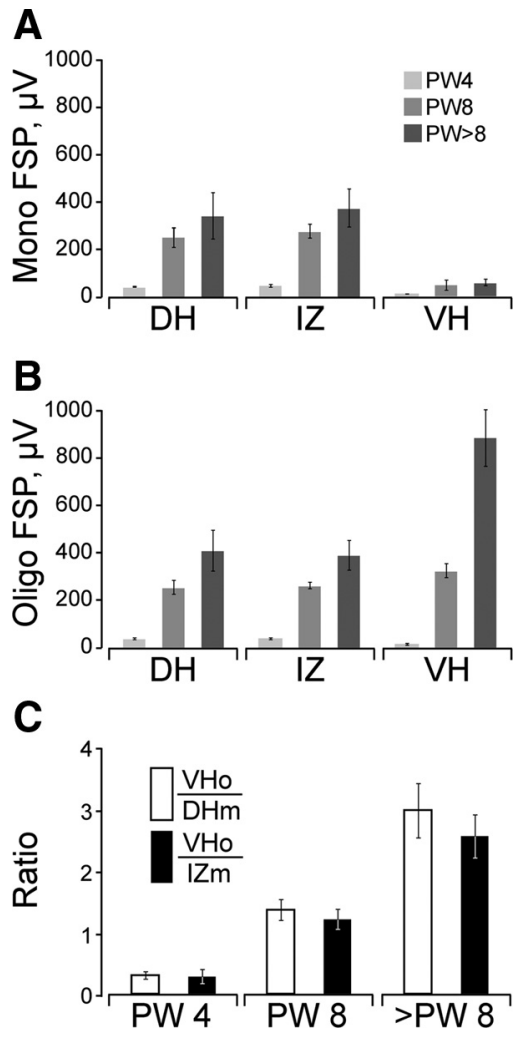

Figure 6. $\quad A, B$, Age-dependent changes in the amplitude of FSPs recorded in the monosynaptic $(\boldsymbol{A})$ and oligosynaptic ranges $(\boldsymbol{B})$. Data are from animals at PW4 (light gray; $n=4)$, PW8 (medium gray; $n=4$ ), and PW11 (dark gray; $n=5$ ). ( plots the ratio of input signals to output. The white bars show the ratios based on dorsal horn monosynaptic-range inputs (white) and intermediate zone inputs (black). All bars plot mean \pm SEM. Statistics are presented in Results. DH, Dorsal horn; IZ, intermediate zone; VH, ventral horn; 0 , oligosynaptic; m, monosynaptic.

PT stimulation consistently increased the likelihood of recording multiunit (and occasionally single unit) activity in PW11 and PW8 animals. Both the tonic and phasic spiking was more prevalent in the ventral horn (supplemental Fig. 3, available at www.jneurosci.org as supplemental material). In contrast, PT stimulation rarely evoked tonic or phasic spiking in PW4 animals. Observations on the activation of spinal-unit activity provide additional support for our hypothesis that CST segmental transmission improves between weeks 5 and 8 .

\section{Unilateral M1 inactivation produces robust ipsilateral CST terminations that activate ventral horn neurons}

M1 inactivation between PW5 and PW7 prevents, contralaterally, the normal maturation of a ChAT phenotype in the intermediate zone and calbindin-positive interneurons in the superficial laminae (Chakrabarty et al., 2009a) and produces a dorsal shift in CST terminations (Martin et al., 2009). These changes in spinal organization of the CS system contralateral to the inactivation are associated with M1 motor map (Chakrabarty et al., 2009b) and motor control impairments (Martin et al., 2000, 2004; Friel et al., 2007). We next asked whether this inactivation also limits development of effective segmental CST transmission to ventral motor circuits. Figure $7 A 1$ shows the distribution of contralateral CST terminations in a representative animal subjected to unilateral M1 inactivation. Terminations, as reported previously (Friel and Martin, 2005, 2007), are dorsal compared with normal development (shown semi-schematically as the dotted outline and shading). $A 2$ and $A 3$ show corresponding spinal recordings contralateral to the inactivated cortex, from the same animal as shown in Figure $7 A 1$. Responses could only be evoked with multiple stimuli in this animal, as well as the other two animals subjected to unilateral inactivation. Multiple stimulus pulses facilitated the postsynaptic component of the CDP (Fig. A2, arrows). Depth recordings to the third pulse are shown in Figure A3ii. A presynaptic volley-like potential was recorded superficially. The amplitude of the potential remained stable for the multiple stimuli, at a given depth. Even with a train of suprathreshold stimuli, only small FSPs are present superficially and none ventrally. For comparison, the dorsoventral distribution of total CST labeling is shown in Figure $7 A 3 i$ (from left to right, low to high local density of CST terminations), adjacent to the depth recordings. The dorsal location of FSPs correlates with the dorsal location of CST terminations. The presence of a volley without large and extensive postsynaptic responses is consistent with a loss of terminations, as reported previously (Friel and Martin, 2005). Because we are examining responses evoked by three stimuli, we cannot be sure whether a response evoked at short latency from the third stimulus is a monosynaptic response or a facilitated oligosynaptic response evoked by the second (or first) stimulus. We also noticed an increase in tonic spiking in the contralateral gray matter after stimulation of the previously silenced PT. However, in contrast to normal-developing PW11 animals, these changes were recorded dorsally, where CST axons terminate, not ventrally (supplemental Fig. 2, available at www.jneurosci.org as supplemental material).

Figure $7 B 1$ shows the ipsilateral CST terminations from the active M1 from the same animal as in A. Because these terminations are ipsilateral to the inactivated $\mathrm{M} 1$, they are terminating on the same side as those shown in A1. These ipsilateral terminations are aberrant; they include transient terminations that are mostly eliminated during normal development. As reported previously (Friel and Martin, 2005), the aberrant ipsilateral terminations overlap minimally with the displaced contralateral CST terminations. Note that the contralateral projections of the active CST have a normal dorsoventral topography (data not shown), as reported previously (Friel and Martin, 2005). For this experiment, we placed a pair of PT stimulating electrodes $\sim 300 \mu \mathrm{m}$ apart to activate one or the other PT during the experiment (supplemental Fig. 1 shows examples of typical PT electrode placements, available at www.jneurosci.org as supplemental material). The amplitude of the contralateral responses were slightly larger than in age-matched controls but not significantly different (monosynaptic: dorsal horn, $F=0.944, p=0.3687$; intermediate zone, $F=2.832$, $p=0.1432$; ventral horn, $F=0.011, p=0.9217$; oligosynaptic: dorsal horn, $F=2.459, p=0.1679$; intermediate zone, $F=2.091, p=0.1983$; ventral horn, $F=0.804, p=0.4044$ ).

The CDP was recorded over the ipsilateral spinal cord (i.e., the side traced in Fig. 7B1 and recorded in B2 and B3). As with stimulation of the previously silenced CST, multiple stimuli facilitated the CDP evoked by stimulation of the active side (Fig. 7B2, arrows). FSPs were recorded from the superficial dorsal horn to the intermediate zone (Fig. 7B3); only the responses evoked by the third stimulus are shown. The pattern of evoked activation was different across the depths, as well as changing locally, from stimulation of the silenced CST. A large positivity was seen after the second and third stimuli (arrow in first trace). This potential was present just underneath the surface and at $500 \mu \mathrm{m}$, was absent at $1 \mathrm{~mm}$, and returned at 1.5 and $2 \mathrm{~mm}$. A delayed negativity was recorded at $1.5 \mathrm{~mm}$ and farther ventrally, within the intermediate zone, in which ipsilateral CST axons terminated (highlighted). The early positivity is likely to reflect a true local outward, inhibitory, current rather than a source for the deeper 
A Silenced CST

A1

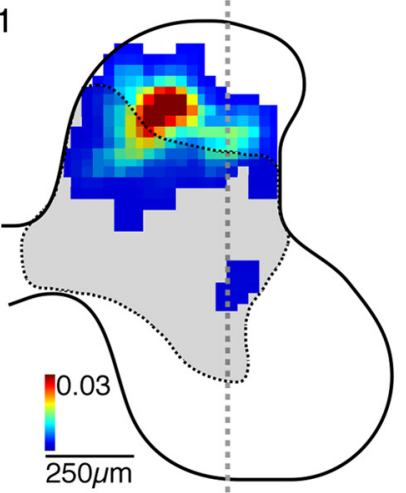

A2

A3

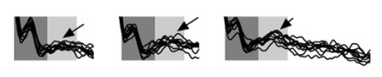

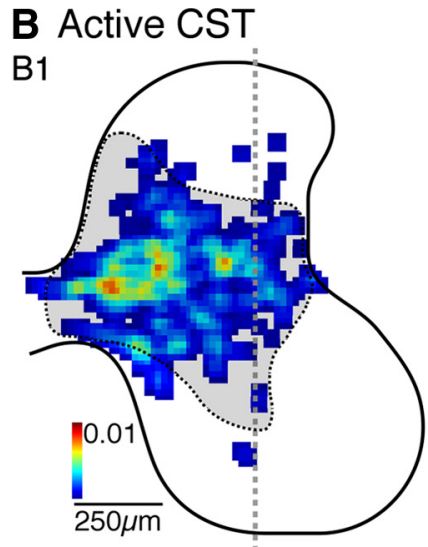

B2

B3 0

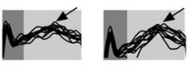

500

1000

1500

2000

2500

3000

3500

4000

4500

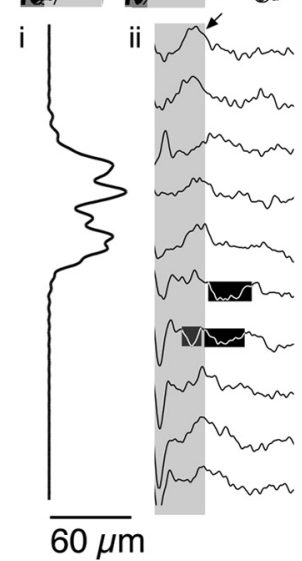

Figure 7. Effect of unilateral inactivation on the distribution of contralateral CST terminations, CDPs, and FSPs from the silenced M1 ( $\boldsymbol{A})$ and the ipsilateral CST terminations, ipsilateral CDPs, and ipsilateral FSPs from the active M1 (B). All data on this figure are from the same animal and the same anatomical side of the cord but either contralateral to the previously silenced $M 1(A)$ or ipsilateral to the initially active M1 $(\boldsymbol{B})$. $\boldsymbol{A} \mathbf{1}, \boldsymbol{B} \mathbf{1}$, Color-coded density maps of CST axon terminations. The color calibration is between 0 and $0.03(\boldsymbol{A} 1)$ or 0.01 (B1) $\mu \mathrm{m}$ axons $/ 30 \mu \mathrm{m}$ square regions. The dashed and shaded region corresponds to the normal contralateral CST termination field. $A \mathbf{2}, \mathbf{B 2}$, CDP from sides of the cord shown in $\boldsymbol{A} \mathbf{1}$ and $\mathbf{B 1}$. $\mathbf{A 3}, \mathbf{B} \mathbf{3}$, Dorsoventral distribution of axonal labeling ( $A 3 i, B 3 i)$ and depth recordings ( $A 3 i i, B 3 i i ;$ depths in micrometers at left). The dorsoventral distributions of axon density were computed from the density maps in $\boldsymbol{A} \mathbf{1}$ and $\boldsymbol{B} 1$. Each line plots the density of axon terminations of the animal, from the dorsal to the ventral borders of the gray mater. Samples were interpolated to 1000 points between the dorsal and ventral margins of the gray matter. Scales are in micrometers of CST axon length within each sequential slice of gray matter. Depth recordings, also from sides of the cord shown in $\boldsymbol{A} \mathbf{1}$ and $\boldsymbol{B} \mathbf{1}$, in response to the third stimulus only. The first two stimuli did not evoke consisting responses. The highlighted areas mark dorsal focal negativities (A3ii) and focal negativities in the intermediate zone (B3ii).

negativity (inward, or excitatory, current). This is because the negativity is later and has a different dorsoventral distribution. It is important to note that we cannot distinguish whether these potentials are in response to the third stimulus or an earlier stimulus. Supplemental Figure 3 (available at www.jneurosci.org as supplemental material) presents ipsilateral recordings from a representative PW11 control animal. Ipsilateral CST terminations were sparse (Theriault and Tatton, 1989; Alisky et al., 1992). Evoked responses were not present, even with stimulation at five times the contralateral threshold ( $60 \mu \mathrm{A}$ threshold; $300 \mu \mathrm{A}$ used).

\section{Projections from the silenced and active CSTs have a} complementary activation pattern in the spinal cord

Comparison of the responses evoked by the previously silenced (Fig. 7A3) and active (Fig. 7B3) sides also reveals a complementary physiology similar to the differential distributions of CST terminations. This is presented in Figure 8 ( $A$ for the animal shown in Fig. 7 and, as average values for the three animals examined, in $B$ ).
When the focal negativity (i.e., excitation) is present in response to stimulation of the previously silenced CST (black line, left), there is the early positive response evoked by stimulation of the active side (i.e., inhibition; gray line, right) at approximately the same depth. As shown in Figure 7, these two potentials have approximately the same latency. As the focal negativity in response to stimulating the previously silenced PT decreased (i.e., the black line transitions closer to zero), the response to stimulating the previously active PT increased (i.e., gray negative line plot becomes larger). These findings show that, because the CST terminations from the two hemispheres are complementary, so too are the effects of stimulation. They further show that the aberrant ipsilateral terminations can activate ventral motor circuits and may thus compensate for the loss of activation of ventral circuits by stimulation of the previously silenced CST.

\section{Discussion}

Development of segmental CST terminations in the cervical gray matter occurs postnatally in the cat and is not complete until approximately PW11-PW14, which is also when the tract and M1 achieve a mature organization (Chakrabarty and Martin, 2000; Li and Martin, 2001; Martin et al., 2009). During this protracted development, there is a brief period when key organizational changes occur to CS system spinal motor circuits. Major refinement of the laterality and the dorsoventral distribution of CST terminations occurs between PW5 and PW7. In this brief period, the CST exerts an activity-dependent trophic influence over maturation of segmental interneuronal circuits, and we postulated that this is critical for the expression of CST motor function (Chakrabarty et al., 2009a). Our results provide physiological support for this hypothesis. Ventral horn oligosynaptic responses become stronger, relative to input responses, at PW8 and older. This shows that segmental circuits become more effective in transmitting CST signals to ventral motor circuits, and possibly motoneurons, during a brief postnatal period.

\section{The importance of segmental corticospinal control}

Ideally, the changing efficacy of spinal transmission of CST signals ought to be studied in awake animals because general anesthesia depresses motor systems function. We used ketamine anesthesia, which results in a more responsive motor system (Di Lazzaro et al., 2003). Increased excitability probably lowered the activation threshold of interneurons, thereby revealing monosynaptic responses with a single PT stimulus pulse, at low strength. In barbiturate-anesthetized preparations, EPSPs are always evoked with at least two pulses at higher strengths (5-20 T) (Illert et al., 1974, 1976). We used weak stimuli (1.5 T) to have a 


\section{A Unilateral FSPs}

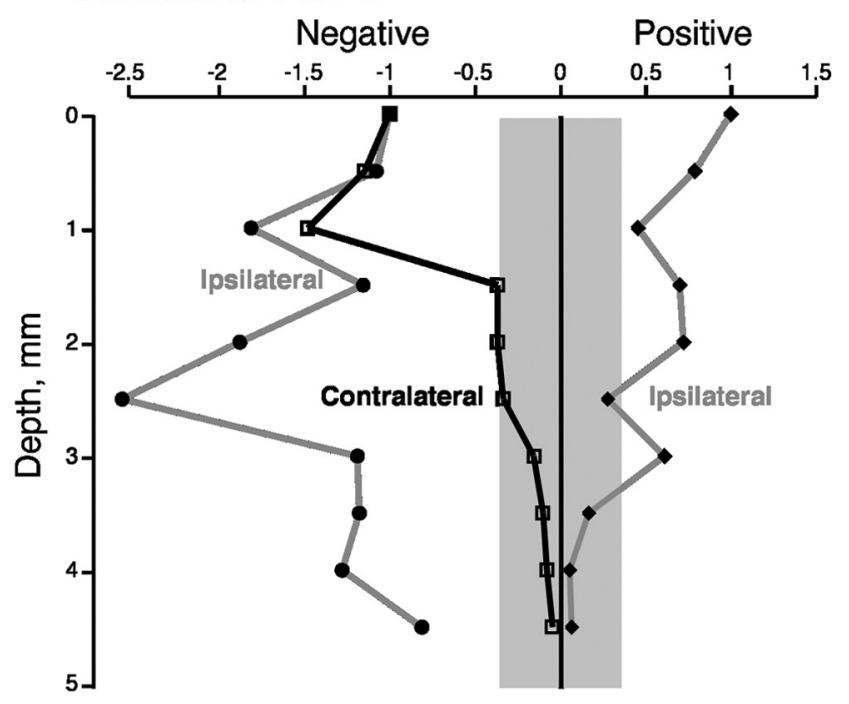

\section{B Average FSPs}

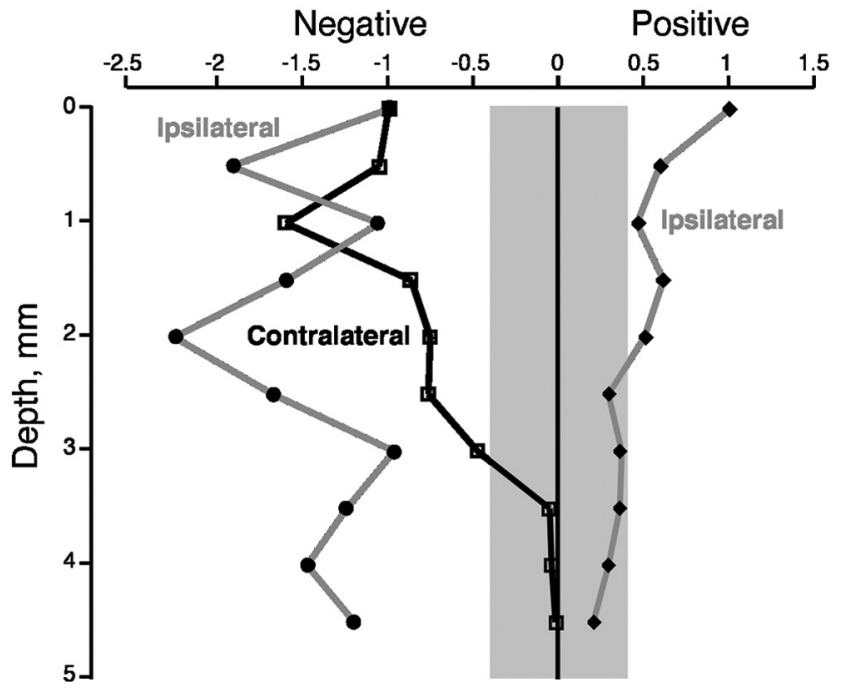

Figure 8. Graphs showing the negative (left) and positive (right) potentials recorded in animals subjected to unilateral $\mathrm{M} 1$ inactivation. All recordings are from the same anatomical side of the spinal cord. Black line plots the negativity recorded contralaterally, in response to stimulation of the PT on the initially silenced side. The gray lines plot positive and negative potentials recorded ipsilaterally, in response to stimulation of the PT on the initially active side. $\boldsymbol{A}$ is a plot of values from the middle electrode penetration in one animal, and $\boldsymbol{B}$ is a plot of the mean of values obtained from three animals (includes the animal shown in $A$ ). The gray band straddling 0 indicates the mean level of random noise recorded along the electrode penetration in $\boldsymbol{A}$ and the mean of the values recorded in three cases in $\boldsymbol{B}$.

highly selective measure of CST activation and spinal transmission, riding on top of an excitable spinal motor circuitry.

We used dorsal horn and intermediate zone monosynaptic responses as a measure of CST input to segmental circuits. This is based on the known anatomy of the CST projections; the densest segmental terminations at all ages are within this region (Armand et al., 1985; Martin, 1996). There are also direct ventral projections outside the motor pools, but these are sparse. The small ventral horn monosynaptic responses (Fig. 7), together with the absence of monosynaptic contacts on motoneurons (Illert et al., 1974; Baldissera et al., 1981), make using the ventral horn oligosynaptic response a good measure of output.
In addition to activating segmental interneurons, PT stimulation activates motoneurons via the $\mathrm{C} 3-\mathrm{C} 4$ propriospinal neurons (PSNs) (Alstermark et al., 2007). Development of this system has not been studied. Although these propriospinal neurons are important for limb control (Alstermark et al., 1981), several findings indicated that segmental transmission is likely to be the major overall factor permitting CST signals to activate ventral motor circuits effectively. First, C3-C4 PSNs are conditionally active during limb movement (Alstermark and Kümmel, 1990b). Second, the densest projection of the CST is to the cervical enlargement in which there is an enormous number of segmental premotor interneurons in the cat (Alstermark and Kümmel, 1990a), many of which are distributed within the CST termination field. Third, an active CST is necessary for development of increased numbers of cholinergic (excitatory) neurons and a relative reduction in the numbers of calbindin neurons, which are likely to be inhibitory (Chakrabarty et al., 2009a).

\section{Role of activity-dependent processes in shaping CST connectivity}

Activity-dependent processes are important in shaping neural circuit development. However, activity manipulations produce aberrant connections that deprive neurons of their appropriate inputs. From the perspective of target circuitry, we are unable to dissociate the postsynaptic activity reduction as a consequence of an activity manipulation from the subsequent connectivity change. This applies to the developing visual as well as the corticospinal system.

After unilateral inactivation of M1, both the silenced CST and the active tract from the other hemisphere develop novel spinal gray matter terminations. The contralateral terminations from the silenced cortex are dorsal and weak. This results in the loss of segmental transmission. The aberrant ipsilateral terminations from the active M1 have a dorsoventral distribution similar to contralateral CST terminations in age-matched controls. Like the silenced CST terminations, they are weak, but a unique response pattern emerged. With multiple stimuli, an early positive, or inhibitory, FSP was evoked, likely mediated mainly by local inhibitory interneurons that receive direct input from CST axons [e.g., calbindin (Fig. 2)] (Illert et al., 1976). Activation of such an aberrant inhibitory circuit could further limit the muted excitatory response evoked by the previously silenced CST. The later negative response from the active CST was largest in the intermediate zone and was present ventrally, suggesting a novel segmental path for segmental transmission of CST signals.

Does the aberrant ipsilateral CST from the active M1 compensate functionally for the loss of strong direct cortical signaling from the inactive M1? This question has important significance for understanding the function of ipsilateral CST connections in health and after trauma (Jankowska and Edgley, 2006; Martin et al., 2009). On one hand, our previous behavioral findings suggest that the aberrant ipsilateral CST axons from the active M1 compensates functionally for the loss of strong direct cortical signaling from the inactive $\mathrm{M} 1$ : reversible inactivation of the previously active M1 produces ipsilateral motor impairments (Martin et al., 2000). On the other hand, after PW5-PW7 inactivation, we are only able to restore normal motor function and a normal M1 motor map with manipulations that lead to a reduction in ipsilateral label and redistribution of the silenced CST terminations to more ventral laminae (Friel and Martin, 2007; Salimi et al., 2008; Chakrabarty et al., 2009b). In the absence of significant activation of ventral motor circuits by the contralateral CST on the affected side of the spinal cord, the aberrant ipsilateral CST 
terminations may be adaptive, because they permit targeted motor responses, albeit with endpoint inaccuracy. However, their presence is not compatible with optimal visuomotor control. That there is an absence of ventral responses in these animals and a persistent motor impairment suggests that spinal circuits contralateral to the inactivated $\mathrm{M} 1$ are not capable of routing the dorsal signals ventrally, showing a clear limitation in the adaptability of local spinal networks.

\section{Mechanisms underlying development of enhanced CST transmission to the ventral horn}

Whereas the CST has a protracted postnatal development, $\sim 3$ months in the cat, our anatomical and physiological findings point to a brief period during which there is a rapid increase in transmission of CS signals from dorsal and intermediate input circuits to ventral output circuits. We refer to this transmission increase as a segmental switch. We propose that this switch enables expression of CST motor functions during postnatal development. There are four complementary mechanisms that could mediate increased efficacy of CS signal transmission to muscle. Each of these potential mechanisms is intrinsic to the spinal segments of the cervical enlargement and are dependent on CS system activity. Impairment in development of any of these potential mechanisms would lead to a segmental block in ventral transmission of CS signals. First, as discussed, there is increased ChAT expression in the principal target interneuron population in the lateral intermediate zone that could be a key component to the increase in CS signaling to the ventral horn and then to muscle (Chakrabarty and Martin, 2009). This ChAT increase depends on CS system activity. Second, CST axon terminal branches develop postnatally, from having few to a large number of preterminal and terminal branches (Li and Martin, 2001). This process is activity and use dependent (Martin et al., 2004; Friel and Martin, 2005). Third, CST presynaptic sites undergo a late postnatal maturation. There are more axonal varicosities that contain synaptophysin at PW8 and older than at PW4 (Meng et al., 2004). This change correlates with improved temporal facilitation of motor output. Fourth, the distribution of early-developing CST axons are not merely diffuse and without connectional specificity. Instead, the distribution of CST axons appears to have a functional logic. Projections to the region containing a subclass of premotor interneuron (i.e., synapse on motoneurons) containing ChAT (Zagoraiou et al., 2009) are absent at PW4 but present at PW8 and older (Chakrabarty et al., 2009a). These interneurons mature early, making effective cholinergic synapses on motoneurons by postnatal days 6-9 in the mouse (Miles et al., 2007), and, in maturity, augment spinal motor output under task-specific conditions (Zagoraiou et al., 2009). All of these developmental events could contribute to closure of the switch to enhance ventral CST transmission. Our findings highlight a novel and important role of spinal interneuronal circuits in enabling the developmental expression of the motor functions of the CST, privileged among the motor pathways as it communicates the most complex and adaptive motor control signals from the cerebral cortex directly to the spinal cord.

\section{References}

Alisky JM, Swink TD, Tolbert DL (1992) The postnatal spatial and temporal development of corticospinal projections in cats. Exp Brain Res 88:265-276.

Alstermark B, Kümmel H (1990a) Transneuronal transport of wheat germ agglutinin conjugated horseradish peroxidase into last order spinal interneurones projecting to acromio- and spinodeltoideus motoneurones in the cat. 1. Location of labelled interneurones and influence of synaptic activity on the transneuronal transport. Exp Brain Res 80:83-95.

Alstermark B, Kümmel H (1990b) Transneuronal transport of wheat germ agglutinin conjugated horseradish peroxidase into last order spinal interneurones projecting to acromio- and spinodeltoideus motoneurones in the cat. 2. Differential labelling of interneurones depending on movement type. Exp Brain Res 80:96-103.

Alstermark B, Lundberg A, Norrsell U, Sybirska E (1981) Integration in the descending motor pathways controlling the forelimb in the cat. 9 . Differential behavioural defects after spinal cord lesion interrupting defined pathways from higher centers to motoneurons. Exp Brain Res 42:299-318.

Alstermark B, Isa T, Pettersson LG, Sasaki S (2007) The C3-C4 propriospinal system in the cat and monkey: a spinal pre-motoneuronal centre for voluntary motor control. Acta Physiol (Oxford) 189:123-140.

Armand J, Holstege G, Kuypers HG (1985) Differential corticospinal projections in the cat. An autoradiographic tracing study. Brain Res 343:351-355.

Baldissera F, Hultborn H, Illert M (1981) Integration in spinal neuronal systems. In: Handbook of physiology, Sect I, The nervous system, Vol II, Motor control (Brooks VB, ed), pp 509-596. Bethesda: American Physiological Society.

Barber RP, Phelps PE, Houser CR, Crawford GD, Salvaterra PM, Vaughn JE (1984) The morphology and distribution of neurons containing choline acetyltransferase in the adult rat spinal cord: an immunocytochemical study. J Comp Neurol 229:329-346.

Barrett P, Bateson P (1978) The development of play in cats. Behaviour 66:106-120.

Brus-Ramer M, Carmel JB, Chakrabarty S, Martin JH (2007) Electrical stimulation of spared corticospinal axons augments connections with ipsilateral spinal motor circuits after injury. J Neurosci 27:13793-13801.

Chakrabarty S, Martin JH (2000) Postnatal development of the motor representation in primary motor cortex. J Neurophysiol 84:2582-2594.

Chakrabarty S, Shulman B, Martin JH (2009a) Activity-dependent codevelopment of the corticospinal system and target interneurons in the cervical spinal cord. J Neurosci 29:8816-8827.

Chakrabarty S, Friel K, Martin JH (2009b) Activity-dependent plasticity improves M1 motor representation and corticospinal tract connectivity. J Neurophysiol 110:1283-1293.

Di Lazzaro V, Oliviero A, Profice P, Pennisi MA, Pilato F, Zito G, Dileone M, Nicoletti R, Pasqualetti P, Tonali PA (2003) Ketamine increases human motor cortex excitability to transcranial magnetic stimulation. J Physiol 547:485-496.

Edgley SA, Jankowska E (1987) Field potentials generated by group II muscle afferents in the middle lumbar segments of the cat spinal cord. J Physiol 385:393-413.

Friel KM, Martin JH (2005) Role of sensory-motor cortex activity in postnatal development of corticospinal axon terminals in the cat. J Comp Neurol 485:43-56.

Friel K, Martin JH (2007) Bilateral activity-dependent interactions in the developing corticospinal system. J Neurosci 27:11083-11090.

Friel KM, Drew T, Martin JH (2007) Differential activity-dependent development of corticospinal control of movement and final limb position during visually-guided locomotion. J Neurophysiol 97:3396-3406.

Illert M, Lundberg A, Tanaka R (1974) Disynaptic corticospinal effects in forelimb motoneurones in the cat. Brain Res 75:312-315.

Illert M, Lundberg A, Tanaka R (1976) Integration in the descending motor pathways controlling the forelimb in the cat. 1. Pyramidal effects on the motoneurons. Exp Brain Res 26:509-519.

Jankowska E, Edgley SA (2006) How can corticospinal tract neurons contribute to ipsilateral movements? A question with implications for recovery of motor functions. Neuroscientist 12:67-79.

Kubota Y, Jones EG (1993) Co-localization of two calcium binding proteins in GABA cells of rat piriform cortex. Brain Res 600:339-344.

Kuypers HGJM (1981) Anatomy of the descending pathways. In: Handbook of physiology, Neurophysiology, Vol II (Brookhart JM, Mountcastle VB, eds), pp 597-666. Bethesda, MD: American Physiological Society.

Li Q, Martin JH (2000) Postnatal development of differential projections from the caudal and rostral motor cortex subregions. Exp Brain Res 134:187-198.

Li Q, Martin JH (2001) Postnatal development of corticospinal axon terminal morphology in the cat. J Comp Neurol 435:127-141. 
Lorente de Nó RA (1947) Action potentials of the motoneurons of the hypoglossus nucleus. J Cell Comp Physiol 29:207-288.

Martin JH (1996) Differential spinal projections from the forelimb areas of the rostral and caudal subregions of primary motor cortex in the cat. Exp Brain Res 108:191-205.

Martin JH, Kably B, Hacking A (1999) Activity-dependent development of cortical axon terminations in the spinal cord and brain stem. Exp Brain Res 125:184-199.

Martin JH, Hacking A, Donarummo L (2000) Impairments in prehension produced by early postnatal sensorimotor cortex activity blockade. J Neurophysiol 83:895-906.

Martin JH, Choy M, Pullman S, Meng Z (2004) Corticospinal development depends on experience. J Neurosci 24:2122-2132.

Martin J, Friel K, Salimi I, Chakrabarty S (2009) Corticospinal development. In: Encyclopedia of neuroscience (Squire L, ed), pp 302-214. Oxford: Academic.

Meng Z, Martin JH (2003) Postnatal development of corticospinal synaptic actions. J Neurophysol 90:683-692.

Meng Z, Li Q, Martin JH (2004) The transition from development to motor control function in the corticospinal system. J Neurosci 24:605-614.

Miles GB, Hartley R, Todd AJ, Brownstone RM (2007) Spinal cholinergic interneurons regulate the excitability of motoneurons during locomotion. Proc Natl Acad Sci U S A 104:2448-2453.
Noga BR, Fortier PA, Kriellaars DJ, Dai X, Detillieux GR, Jordan LM (1995) Field potential mapping of neurons in the lumbar spinal cord activated following stimulation of the mesencephalic locomotor region. J Neurosci 15:2203-2217.

Porter R, Lemon R (1993) Corticospinal function and voluntary movement. Oxford: Oxford Science.

Prechtl HFR (1997) The importance of fetal movements. In: Neurophysiology and neuropsychology of motor development (Connolly KJ, Forssberg H, eds), pp 42-53. London: MacKeith.

Salimi I, Friel K, Martin JH (2008) Pyramidal tract stimulation restores normal corticospinal tract connections and visuomotor skill after early postnatal motor cortex activity blockade. J Neurosci 28:7426-7434.

Theriault E, Tatton WG (1989) Postnatal redistribution of pericruciate motor cortical projections within the kitten spinal cord. Dev Brain Res 45:219-237.

Villablanca JR, Olmstead CE (1979) Neurological development of kittens. Dev Psychol 12:101-127.

Weidner N, Ner A, Salimi N, Tuszynski MH (2001) Spontaneous corticospinal axonal plasticity and functional recovery after adult central nervous system injury. Proc Natl Acad Sci U S A 98:3513-3518.

Zagoraiou L, Akay T, Martin JF, Brownstone RM, Jessell TM, Miles GM (2009) A cluster of cholinergic premotor interneurons modulates mouse locomotor activity. Neuron 64:645-662. 